\title{
Certain Inequalities of Multivalent Analytic Functions with Missing Coefficients
}

\author{
Yi-Ling Cang ${ }^{1}$ and Cai-Mei Yan $^{2}$ \\ ${ }^{1}$ Department of Mathematics, Suqian College, Suqian 223800, China \\ ${ }^{2}$ Information Engineering College, Yangzhou University, Yangzhou 225009, China
}

Correspondence should be addressed to Cai-Mei Yan, cmyan@yzu.edu.cn

Received 22 November 2012; Accepted 31 December 2012

Academic Editor: Jacek Dziok

Copyright (c) 2013 Y.-L. Cang and C.-M. Yan. This is an open access article distributed under the Creative Commons Attribution License, which permits unrestricted use, distribution, and reproduction in any medium, provided the original work is properly cited.

The purpose of the present paper is to derive the radius of starlikeness for certain $p$-valent functions with missing coefficients. The results obtained here are sharp.

\section{Introduction}

Let $A_{p}(n)$ denote the class of functions of the form

$$
f(z)=z^{p}+\sum_{k=n}^{\infty} a_{p+k} z^{p+k} \quad(p, n \in N=\{1,2,3, \ldots\}),
$$

which are $p$-valent analytic in the open unit disk $U=\{z$ : $z \in C$ and $|z|<1\}$. A function $f(z) \in A_{p}(n)$ is said to be $p$-valently starlike of order $\rho$ in $U$ if it satisfies

$$
\operatorname{Re}\left(\frac{z f^{\prime}(z)}{f(z)}\right)>\rho \quad(0 \leq \rho<p ; z \in U) .
$$

For functions $f(z)$ and $g(z)$ analytic in $U$, we say that $f(z)$ is subordinate to $g(z)$ in $U$, and we write $f(z) \prec g(z)(z \in U)$, if there exists an analytic function $w(z)$ in $U$ such that

$$
|w(z)| \leq|z|, \quad f(z)=g(w(z)) \quad(z \in U) .
$$

Furthermore, if the function $g(z)$ is univalent in $U$, then

$$
\begin{gathered}
f(z) \prec g(z) \quad(z \in U) \Longleftrightarrow f(0)=g(0), \\
f(U) \subset g(U) .
\end{gathered}
$$

A number of results for $p$-valently starlike functions have been obtained by several authors (see, e.g., [1-7]). In this note, we shall derive the radius of starlikeness for certain $p$-valent functions with missing coefficients.

\section{Main Results}

Our main result is the following.

Theorem 1. Let $f(z)$ belong to the class $A_{p}(n)$ and satisfy

$$
\frac{f(z)}{z^{p}} \prec\left(\frac{1+z}{1-z}\right)^{\gamma} \quad(0<\gamma \leq 1 ; z \in U) .
$$

Then,

$$
\begin{array}{r}
\operatorname{Re}\left\{(1-\delta)\left(\frac{f(z)}{z^{p}}\right)^{1 / \gamma}+\delta \frac{z f^{\prime}(z)}{f(z)}\right\}>\rho \\
\left(|z|<r_{n}(p, \gamma, \delta, \rho)\right),
\end{array}
$$

where $0<\delta \leq 1,0 \leq \rho<1$ and $r_{n}(p, \gamma, \delta, \rho)$ is the smallest root in $(0,1)$ of the equation

$$
\begin{gathered}
{[1+\rho-(p+1) \delta] r^{2 n}-2(1-\delta+n \delta \gamma) r^{n}} \\
+1-\rho+(p-1) \delta=0 .
\end{gathered}
$$

The result is sharp.

Proof. From (5), we can write that

$$
\left(\frac{f(z)}{z^{p}}\right)^{1 / \gamma}=\frac{1+z^{n} \varphi(z)}{1-z^{n} \varphi(z)}
$$


where $\varphi(z)$ is analytic and $|\varphi(z)| \leq 1$ in $U$. Differentiating both sides of (8) logarithmically, we arrive at

$$
\frac{z f^{\prime}(z)}{f(z)}=p+\frac{2 n \gamma z^{n} \varphi(z)}{1-\left(z^{n} \varphi(z)\right)^{2}}+\frac{2 \gamma z^{n+1} \varphi^{\prime}(z)}{1-\left(z^{n} \varphi(z)\right)^{2}} \quad(z \in U) .
$$

Put $|z|=r<1$ and $\left(f(z) / z^{p}\right)^{1 / \gamma}=u+i v(u, v \in R)$. Then, (8) implies that

$$
\begin{aligned}
& z^{n} \varphi(z)=\frac{u-1+i v}{u+1+i v} \\
& \frac{1-r^{n}}{1+r^{n}} \leq u \leq \frac{1+r^{n}}{1-r^{n}}
\end{aligned}
$$

With the help of the Carathéodory inequality:

$$
\left|\varphi^{\prime}(z)\right| \leq \frac{1-|\varphi(z)|^{2}}{1-r^{2}}
$$

it follows from (10) that

$$
\begin{aligned}
& \operatorname{Re}\{(\left.(-\delta)\left(\frac{f(z)}{z^{p}}\right)^{1 / \gamma}+\delta \frac{z f^{\prime}(z)}{f(z)}\right\} \\
& \geq(1-\delta) u+p \delta+2 n \delta \gamma \operatorname{Re}\left\{\frac{z^{n} \varphi(z)}{1-\left(z^{n} \varphi(z)\right)^{2}}\right\} \\
&-2 \delta \gamma\left|\frac{z^{n+1} \varphi^{\prime}(z)}{1-\left(z^{n} \varphi(z)\right)^{2}}\right| \\
& \geq(1-\delta) u+p \delta+\frac{n \delta \gamma}{2}\left(u-\frac{u}{u^{2}+v^{2}}\right) \\
&+\frac{\delta \gamma}{2} \frac{(u-1)^{2}+v^{2}-r^{2 n}\left((u+1)^{2}+v^{2}\right)}{r^{n-1}\left(1-r^{2}\right)\left(u^{2}+v^{2}\right)^{1 / 2}} \\
&= F_{n}(u, v) \quad(\text { say }), \\
& \frac{\partial}{\partial v} F_{n}(u, v)=\delta \gamma v G_{n}(u, v),
\end{aligned}
$$

where $0<r<1,0<\delta \leq 1$ and

$$
\begin{aligned}
G_{n}(u, v)= & \frac{n u}{\left(u^{2}+v^{2}\right)^{2}}+\frac{1-r^{2 n}}{r^{n-1}\left(1-r^{2}\right)\left(u^{2}+v^{2}\right)^{1 / 2}} \\
& +\frac{r^{2 n}\left((u+1)^{2}+v^{2}\right)-\left((u-1)^{2}+v^{2}\right)}{2 r^{n-1}\left(1-r^{2}\right)\left(u^{2}+v^{2}\right)^{3 / 2}} .
\end{aligned}
$$

From (10), we can see that

$$
r^{2 n}\left((u+1)^{2}+v^{2}\right)-\left((u-1)^{2}+v^{2}\right) \geq 0 .
$$

Thus, we have from (10), (11), and (15) that

$$
G_{n}(u, v)>0 .
$$

Since $F_{n}(u, v)$ is a even function of $v$, from (13), (14), and (15), we see that

$$
\begin{aligned}
F_{n}(u, v) \geq & F_{n}(u, 0) \\
= & (1-\delta) u+p \delta+\frac{n \delta \gamma}{2}\left(u-\frac{1}{u}\right) \\
& +\frac{\delta \gamma}{2 r^{n-1}\left(1-r^{2}\right)} \\
& \times\left[\left(1-r^{2 n}\right)\left(u+\frac{1}{u}\right)-2\left(1+r^{2 n}\right)\right] .
\end{aligned}
$$

Let us now calculate the minimum value of $F_{n}(u, 0)$ on the closed interval $\left[\left(1-r^{n}\right) /\left(1+r^{n}\right),\left(1+r^{n}\right) /\left(1-r^{n}\right)\right]$. Noting that

$$
\frac{1-r^{2 n}}{r^{n-1}\left(1-r^{2}\right)} \geq n
$$

(see [8]) and lower bound in (11), we deduce from (18) that

$$
\begin{aligned}
\frac{d}{d u} F_{n}(u, 0)= & -\delta \\
& +\frac{\delta \gamma}{2}\left[\left(\frac{1-r^{2 n}}{r^{n-1}\left(1-r^{2}\right)}+n\right)\right. \\
& \left.\quad-\frac{1}{u^{2}}\left(\frac{1-r^{2 n}}{r^{n-1}\left(1-r^{2}\right)}-n\right)\right] \\
\geq & +\frac{\delta \gamma}{2}\left[\left(\frac{1-r^{2 n}}{r^{n-1}\left(1-r^{2}\right)}+n\right)\right. \\
& \left.\quad-\left(\frac{1+r^{n}}{1-r^{n}}\right)^{2}\left(\frac{1-r^{2 n}}{r^{n-1}\left(1-r^{2}\right)}-n\right)\right] \\
= & 1-\delta+\frac{2 \delta \gamma I_{n}(r)}{\left(1-r^{n}\right)^{2}},
\end{aligned}
$$

where

$$
I_{n}(r)=\frac{n}{2}\left(1+r^{2 n}\right)-r\left(1+r^{2}+\cdots+r^{2 n-2}\right) .
$$

Also,

$$
I_{n}^{\prime}(r)=n^{2} r^{2 n-1}-\left(1+3 r^{2}+\cdots+(2 n-1) r^{2 n-2}\right)
$$

$I_{1}^{\prime}(r)=r-1<0$. Suppose that $I_{n}^{\prime}(r)<0$. Then,

$$
\begin{aligned}
I_{n+1}^{\prime}(r)= & (n+1)^{2} r^{2 n+1}-(2 n+1) r^{2 n} \\
& -\left(1+3 r^{2}+\cdots+(2 n-1) r^{2 n-2}\right) \\
< & n^{2} r^{2 n}-\left(1+3 r^{2}+\cdots+(2 n-1) r^{2 n-2}\right) \\
< & I_{n}^{\prime}(r)<0 .
\end{aligned}
$$


Hence, by virtue of the mathematical induction, we have $I_{n}^{\prime}(r)<0$ for all $n \in N$ and $0 \leq r<1$. This implies that

$$
I_{n}(r)>I_{n}(1)=0 \quad(n \in N ; 0 \leq r<1) .
$$

In view of (20) and (24), we see that when $u \in\left[\left(1-r^{n}\right) /(1+\right.$ $\left.\left.r^{n}\right),\left(1+r^{n}\right) /\left(1-r^{n}\right)\right]$, then

$$
\frac{d}{d u} F_{n}(u, 0)>0
$$

Further, it follows from (13), (18), and (25) that

$$
\begin{aligned}
\operatorname{Re}\{ & \left.(1-\delta)\left(\frac{f(z)}{z^{p}}\right)^{1 / \gamma}+\delta \frac{z f^{\prime}(z)}{f(z)}\right\}-\rho \\
& \geq F_{n}\left(\frac{1-r^{n}}{1+r^{n}}, 0\right)-\rho \\
& =(1-\delta) \frac{1-r^{n}}{1+r^{n}}+\delta \frac{p-2 n \gamma r^{n}-p r^{2 n}}{1-r^{2 n}}-\rho \\
& =\frac{J_{n}(r)}{1-r^{2 n}},
\end{aligned}
$$

where $0 \leq \rho<1$ and

$$
\begin{aligned}
J_{n}(r)= & {[1+\rho-(p+1) \delta] r^{2 n}-2(1-\delta+n \delta \gamma) r^{n} } \\
& +1-\rho+(p-1) \delta .
\end{aligned}
$$

Note that $J_{n}(0)=1-\rho+(p-1) \delta>0$ and $J_{n}(1)=-2 n \delta \gamma<0$. If we let $r_{n}(p, \gamma, \delta, \rho)$ denote the smallest root in $(0,1)$ of the equation $J_{n}(r)=0$, then (26) yields the desired result (6).

To see that the bound $r_{n}(p, \gamma, \delta, \rho)$ is the best possible one, we consider the function

$$
f(z)=z^{p}\left(\frac{1+z^{n}}{1-z^{n}}\right)^{\gamma}
$$

It is clear that for $z=r \in\left(r_{n}(p, \gamma, \delta, \rho), 1\right)$,

$$
(1-\delta)\left(\frac{f(r)}{r^{p}}\right)^{1 / \gamma}+\delta \frac{r f^{\prime}(r)}{f(r)}-\rho=\frac{J_{n}(r)}{1-r^{2 n}}<0,
$$

which shows that the bound $r_{n}(p, \gamma, \delta, \rho)$ cannot be increased.

Setting $\delta=1$, Theorem 1 reduces to the following result which gives the radius of starlikeness for certain $p$-valent analytic functions with missing coefficients.

Corollary 2. Let $f(z)$ satisfy the condition (5) and $0 \leq \rho<1$. Then, $f(z)$ is starlike of order $\rho$ in

$$
|z|<\left[\frac{\left((n \gamma)^{2}+(p-\rho)^{2}\right)^{1 / 2}-n \gamma}{p-\rho}\right]^{1 / n} .
$$

\section{Acknowledgment}

The authors would like to express sincere thanks to the referees for careful reading and suggestions which helped them to improve the paper.

\section{References}

[1] J.-L. Liu and H. M. Srivastava, "Some convolution conditions for starlikeness and convexity of meromorphically multivalent functions," Applied Mathematics Letters, vol. 16, no. 1, pp. 13-16, 2003.

[2] M. Nunokawa, "On the order of strongly starlikeness of strongly convex functions," Proceedings of the Japan Academy A, vol. 69, no. 7, pp. 234-237, 1993.

[3] M. Nunokawa, S. Owa, H. Saitoh, A. Ikeda, and N. Koike, "Some results for strongly starlike functions," Journal of Mathematical Analysis and Applications, vol. 212, no. 1, pp. 98-106, 1997.

[4] M. Nunokawa and D. K. Thomas, "On convex and starlike functions in a sector," Australian Mathematical Society A, vol. 60, no. 3, pp. 363-368, 1996.

[5] M. Obradović and S. Owa, "Some sufficient conditions for strongly starlikeness," International Journal of Mathematics and Mathematical Sciences, vol. 24, no. 9, pp. 643-647, 2000.

[6] N. Takahashi and M. Nunokawa, "A certain connection between starlike and convex functions," Applied Mathematics Letters, vol. 16, no. 5, pp. 653-655, 2003.

[7] N. Xu and D.-G. Yang, "An application of differential subordinations and some criteria for starlikeness," Indian Journal of Pure and Applied Mathematics, vol. 36, no. 10, pp. 541-556, 2005.

[8] D.-G. Yang and J.-L. Liu, "On a class of analytic functions with missing coefficients," Applied Mathematics and Computation, vol. 215, no. 9, pp. 3473-3481, 2010.

The result is sharp. 


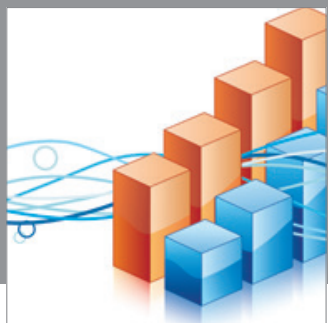

Advances in

Operations Research

mansans

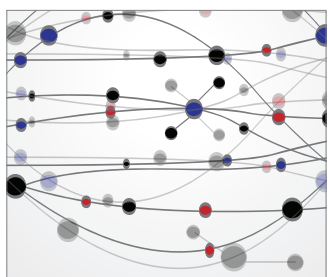

The Scientific World Journal
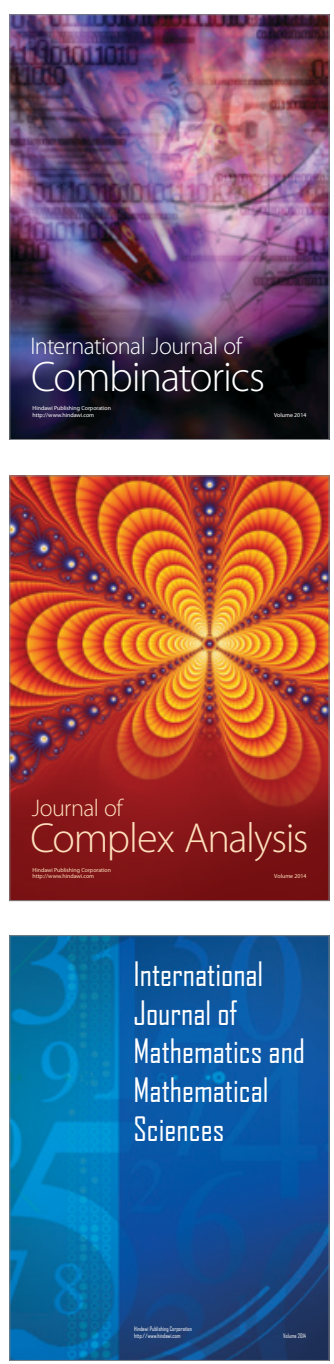
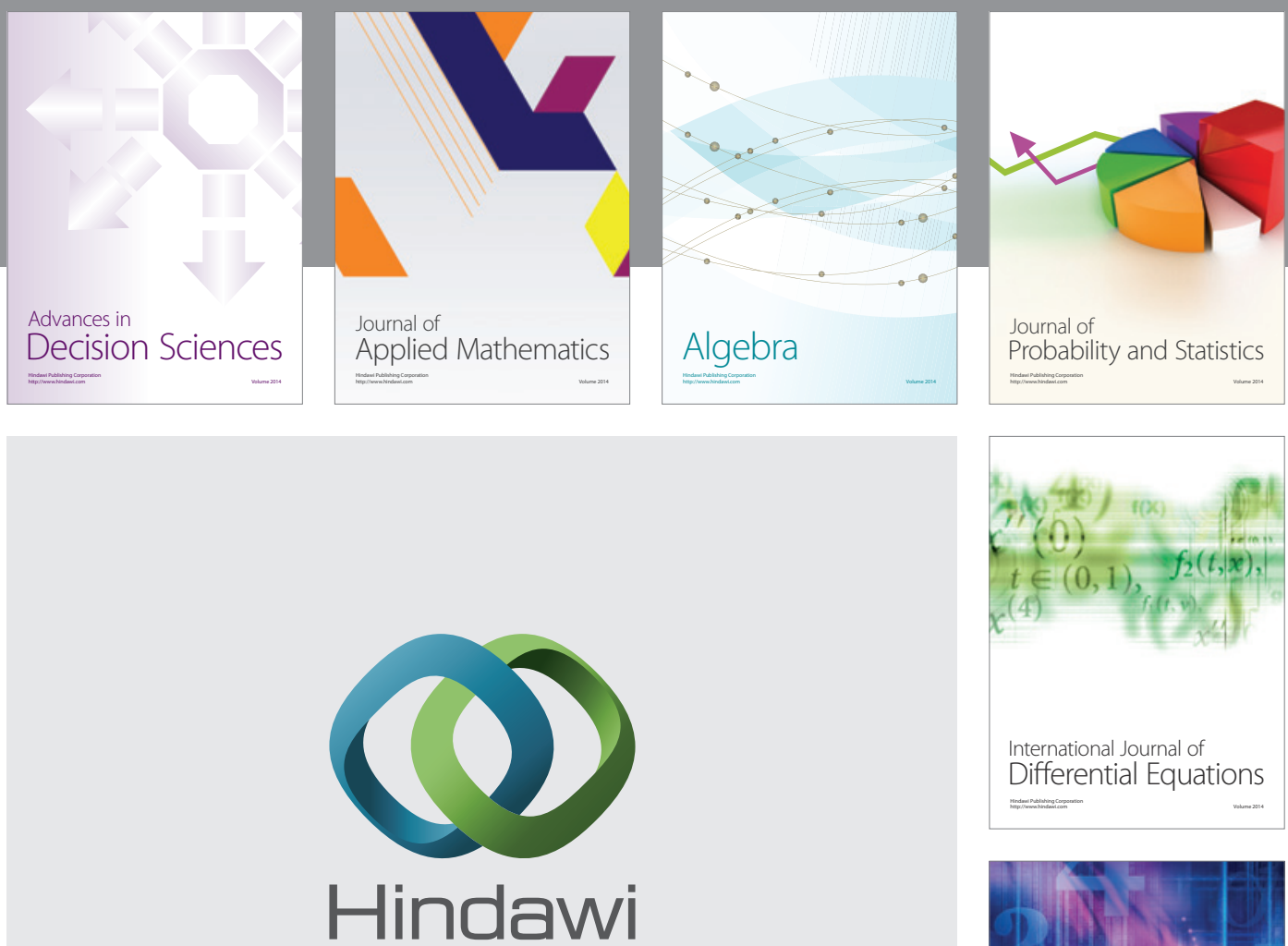

Submit your manuscripts at http://www.hindawi.com
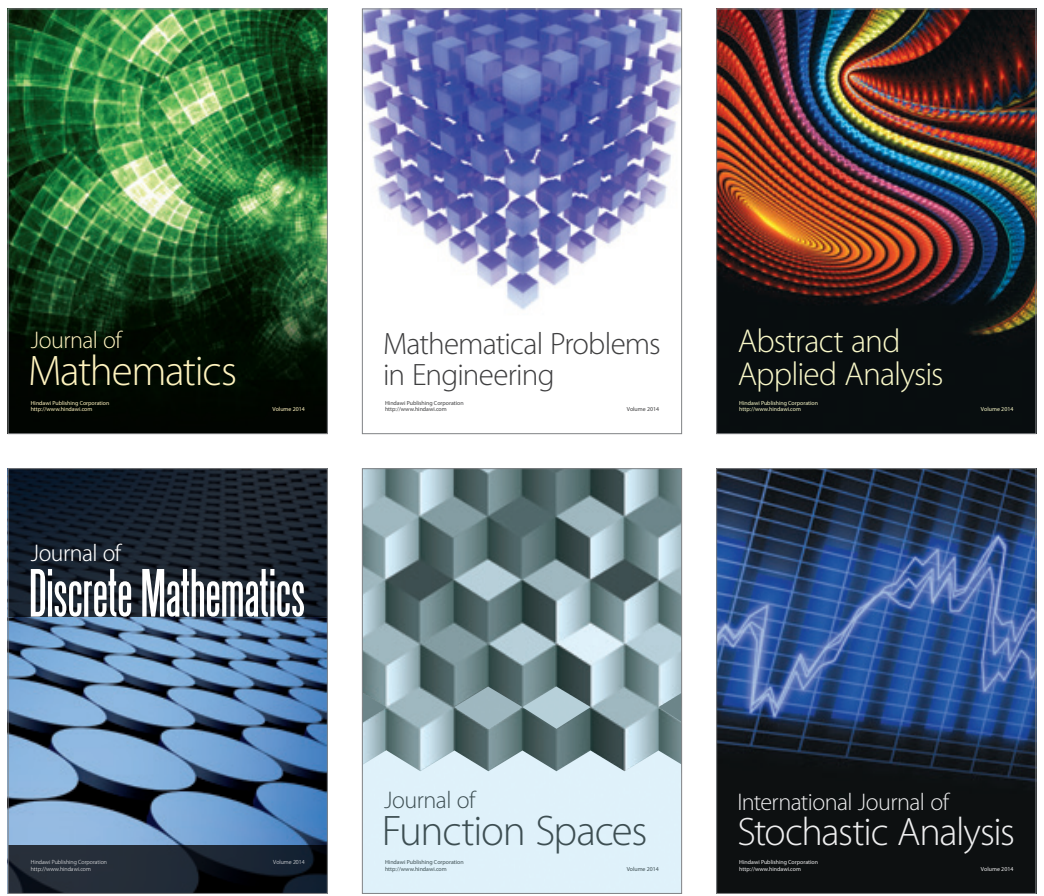

Journal of

Function Spaces

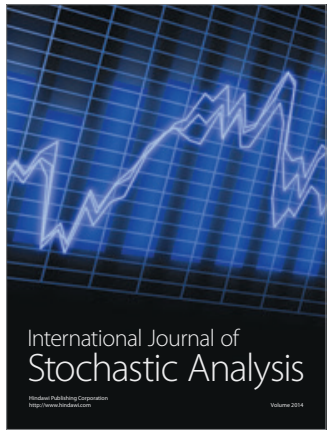

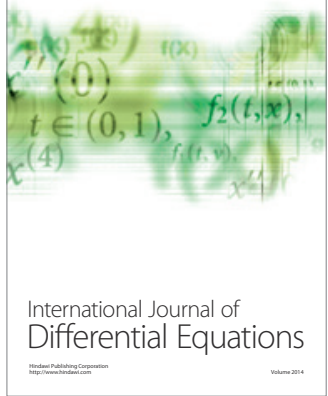
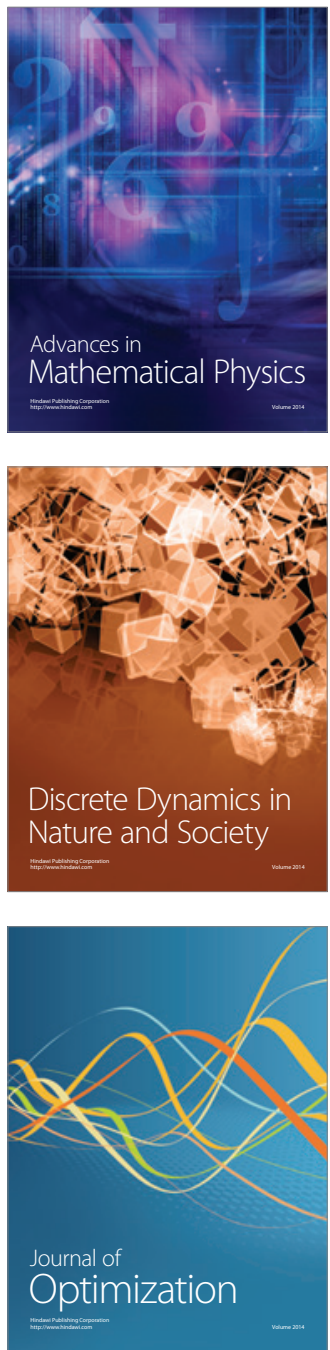